\title{
A Third Vesicular Glutamate Transporter Expressed by Cholinergic and Serotoninergic Neurons
}

\author{
Christelle Gras, ${ }^{1,2 *}$ Etienne Herzog, ${ }^{1 *}$ Gian Carlo Bellenchi, ${ }^{2}$ Véronique Bernard, ${ }^{3}$ Philippe Ravassard, ${ }^{4}$ \\ Michel Pohl, ${ }^{5}$ Bruno Gasnier, ${ }^{2}$ Bruno Giros, ${ }^{1}$ and Salah El Mestikawy ${ }^{1}$ \\ ${ }^{1}$ Faculté de Médecine, Institut National de la Santé et de la Recherche Médicale (INSERM) Unité 513, 94010 Créteil \\ Cedex, France, 2Institut de Biologie Physico-Chimique, Centre National de la Recherche Scientifique (CNRS) Unité Propre \\ de Recherche 1929, 75005 Paris, France, ${ }^{3}$ CNRS Unité Mixte de Recherche (UMR) 5541, Université Bordeaux 2, 33076 \\ Bordeaux Cedex, France, ${ }^{4}$ CNRS UMR 7091, Hôpital Pitié-Salpétrière, 75013 Paris, France, and 5 Faculté de Médecine \\ Pitié Salpétrière, INSERM Unité 288, 75013 Paris, France
}

Two proteins previously known as $\mathrm{Na}^{+}$-dependent phosphate transporters have been identified recently as vesicular glutamate transporters (VGLUT1 and VGLUT2). Together, VGLUT1 and VGLUT2 are operating at most central glutamatergic synapses. In this study, we characterized a third vesicular glutamate transporter (VGLUT3), highly homologous to VGLUT1 and VGLUT2. Vesicles isolated from endocrine cells expressing recombinant VGLUT3 accumulated L-glutamate with bioenergetic and pharmacological characteristics similar, but not identical, to those displayed by the type-1 and type- 2 isoforms. Interestingly, the distribution of VGLUT3 mRNA was restricted to a small number of neurons scattered in the striatum, hippocampus, cerebral cortex, and raphe nuclei, in contrast to
VGLUT1 and VGLUT2 transcripts, which are massively expressed in cortical and deep structures of the brain, respectively. At the ultrastructural level, VGLUT3 immunoreactivity was concentrated over synaptic vesicle clusters present in nerve endings forming asymmetrical as well as symmetrical synapses. Finally, VGLUT3-positive neurons of the striatum and raphe nuclei were shown to coexpress acetylcholine and serotonin transporters, respectively. Our study reveals a novel class of glutamatergic nerve terminals and suggests that cholinergic striatal interneurons and serotoninergic neurons from the brainstem may store and release glutamate.

Key words: glutamate; VGLUT3; neurotransmitter transporter; synaptic vesicle; excitatory neurotransmission; brain
Glutamate, a neurotransmitter used by a majority of excitatory connections in the mammalian brain, has to be loaded into synaptic vesicles by proton-dependent transporters before its exocytotic release (Ozkan and Ueda, 1998; Reimer et al., 1998; Erickson and Varoqui, 2000; Gasnier, 2000). Brain-specific $\mathrm{Na}^{+}$dependent inorganic phosphate transporter (Ni et al., 1994) and differentiation-associated $\mathrm{Na}^{+}$-dependent inorganic phosphate transporter (Aihara et al., 2000), two members of the $\mathrm{Na}^{+}$dependent inorganic phosphate transporter family, are now unambiguously established as two vesicular glutamate transporters (VGLUT1 and VGLUT2) by an array of biochemical, anatomical, electrophysiological, and genetic evidence (Dent et al., 1997; Lee et al., 1999; Bellocchio et al., 2000; Takamori et al., 2000, 2001; Bai et al., 2001; Fremeau et al., 2001; Herzog et al., 2001). Both transporters are abundantly expressed in the brain (Ni et al., 1995; Hisano et al., 1997). VGLUT1 is massively present in excitatory neurons from the cerebral and cerebellar cortices, as well as the hippocampus, whereas most glutamatergic neurons from the di-

\footnotetext{
Received Feb. 12, 2002; revised April 15, 2002; accepted April 18, 2002.

*C.G. and E.H. contributed equally to this work.

This research was supported by grants from Hoescht Marion Roussel and Institut National de la Santé et de la Recherche Médicale (B. Giros) and Centre National de la Recherche Scientifique. G.C.B was supported by the European Community Training and Mobility for Researchers Program (contract no. FMRX-CT98-0228). We thank B. Wiedenmann for giving the BON cell line and E. Doudnikoff for technical support on electronic microscopy. We are also very grateful to P. Ascher, J. P. Henry, and C. Mulle for their helpful advice and discussion.

Correspondence should be addressed to Dr. Salah El Mestikawy, Faculté de Médecine, Institut National de la Santé et de la Recherche Médicale Unité 513, 8 rue du Général Sarrail, 94010 Créteil Cedex, France. E-mail: salah.elmestikawy@im3. inserm.fr.

Copyright (C) 2002 Society for Neuroscience $\quad 0270-6474 / 02 / 225442-10 \$ 15.00 / 0$
}

encephalon and rhombencephalon preferentially use VGLUT2 (Fremeau et al., 2001; Herzog et al., 2001; Varoqui et al., 2002). At the subcellular level, VGLUT1 and VGLUT2 are found in synaptic vesicles located in terminals forming asymmetrical contacts (Bellocchio et al., 1998; Fremeau et al., 2001; Fujiyama et al., 2001; Hayashi et al., 2001; Sakata-Haga et al., 2001; Takamori et al., 2001; Varoqui et al., 2002), the hallmark of glutamatergic terminals (Shepherd, 1998). Together, VGLUT1 and VGLUT2, with their complementary distributions, seem to account for most of the known glutamatergic neurons of the CNS (Fremeau et al., 2001; Varoqui et al., 2002).

We now report the isolation of VGLUT3, an unforeseen third vesicular glutamate transporter. Although structurally and functionally closely related to the widely expressed VGLUT1 and VGLUT2, this novel transporter exhibits several unique features. In particular, it is found in all cholinergic interneurons of the striatum, as well as in serotoninergic neurons from the raphe nuclei.

\section{MATERIALS AND METHODS}

cDNA cloning and expression in BON cells. VGLUT3 full-length clones were obtained by screening a $\lambda$ Zap II rat hippocampus cDNA library (Stratagene, La Jolla, CA) as described previously (Herzog et al., 2001). Inserts were sequenced and subcloned into the expression vector pcDNA3 (Invitrogen, San Diego, CA).

The neuroendocrine cell line BON (Everest et al., 1991) (a kind gift from B. Wiedenmann, Humboldt University, Berlin, Germany) was cultured at $37^{\circ} \mathrm{C}$ under $5 \% \mathrm{CO}_{2}$ in DMEM/nutrient mix F-12 (1:1) (Invitrogen), supplemented with $7.5 \%$ fetal bovine serum, $100 \mathrm{U} / \mathrm{ml}$ penicillin, and $100 \mu \mathrm{g} / \mathrm{ml}$ streptomycin. After electroporation with the pcDNA3VGLUT3 plasmid, stable transfectants were selected in the presence of 
$800 \mu \mathrm{g} / \mathrm{ml} \mathrm{G} 418$ (Invitrogen), screened by immunofluorescence with a serum against a VGLUT3 peptide (P45-1; see below) and further confirmed using a serum raised to another peptide (P45-3; see below). BON cells stably transfected with rat VGLUT2 or vesicular inhibitory amino acid transporter (VIAAT) cDNAs were used as controls (Herzog et al., 2001).

Amino acid uptake assay. For each BON transfectant, cells from four confluent $15 \mathrm{~cm}$ dishes $\left(\sim 150 \times 10^{6}\right.$ cells $)$ were washed once with PBS, scrapped, and recovered in $10 \mathrm{ml}$ of chilled $0.32 \mathrm{M}$ sucrose and $4 \mathrm{~mm}$ HEPES-KOH, $\mathrm{pH}$ 7.4. Cells were broken using a Bioneb cell disruption system (Glas-Gol, Terre Haute, IN), with a single cycle of atomization under a $3.3 \mathrm{l} / \mathrm{min}$ stream of nitrogen at 2.5 bar. Organelle intactness was assessed by measuring the latency of a lysosomal enzyme, $\beta$-glucuronidase, using 4-methylumbelliferyl- $\beta$-D-glucuronide as a substrate. Typically, $\sim 60 \%$ of $\beta$-glucuronidase activity was protected in the absence of Triton X-100, showing that an identical percentage of lysosomes remained intact after cell breakage. Nuclei and cell debris were discarded by centrifugation at $10,000 \times g$ for $5 \mathrm{~min}$. Membranes were then pelleted at $200,000 \times g$ for $20 \mathrm{~min}$ and resuspended in $400 \mu \mathrm{l}$ of ice-cold $0.32 \mathrm{~m}$ sucrose, $4 \mathrm{~mm} \mathrm{KCl,} 4 \mathrm{~mm} \mathrm{MgSO}_{4}$, and $10 \mathrm{~mm}$ HEPES$\mathrm{KOH}, \mathrm{pH} 7.4$ (uptake buffer).

The transport reaction was started by addition of $10 \mu \mathrm{l}$ of membranes (100 $\mu \mathrm{g}$ of protein) to $90 \mu \mathrm{l}$ of uptake buffer containing $2.2 \mathrm{~mm} \mathrm{ATP,} 1.1$ $\mathrm{mM} \mathrm{MgSO}_{4}$, and $44 \mu \mathrm{M}(2 \mu \mathrm{Ci})\left[{ }^{3} \mathrm{H}\right] \mathrm{L}$-glutamate or $\left[{ }^{3} \mathrm{H}\right] \mathrm{L}$-aspartate (both from Amersham Biosciences, Buckinghamshire, UK), with or without 50 $\mu \mathrm{M}$ carbonyl cyanide m-chlorophenylhydrazone (CCCP) and other additives, as stated in Results and figure legends. After incubation at $30^{\circ} \mathrm{C}$ for $10 \mathrm{~min},\left[{ }^{3} \mathrm{H}\right]$ amino acid uptake was terminated by dilution with $3 \mathrm{ml}$ of ice-cold $0.15 \mathrm{M} \mathrm{KCl}$, rapid filtration through a $0.45 \mu \mathrm{m}$ pore size

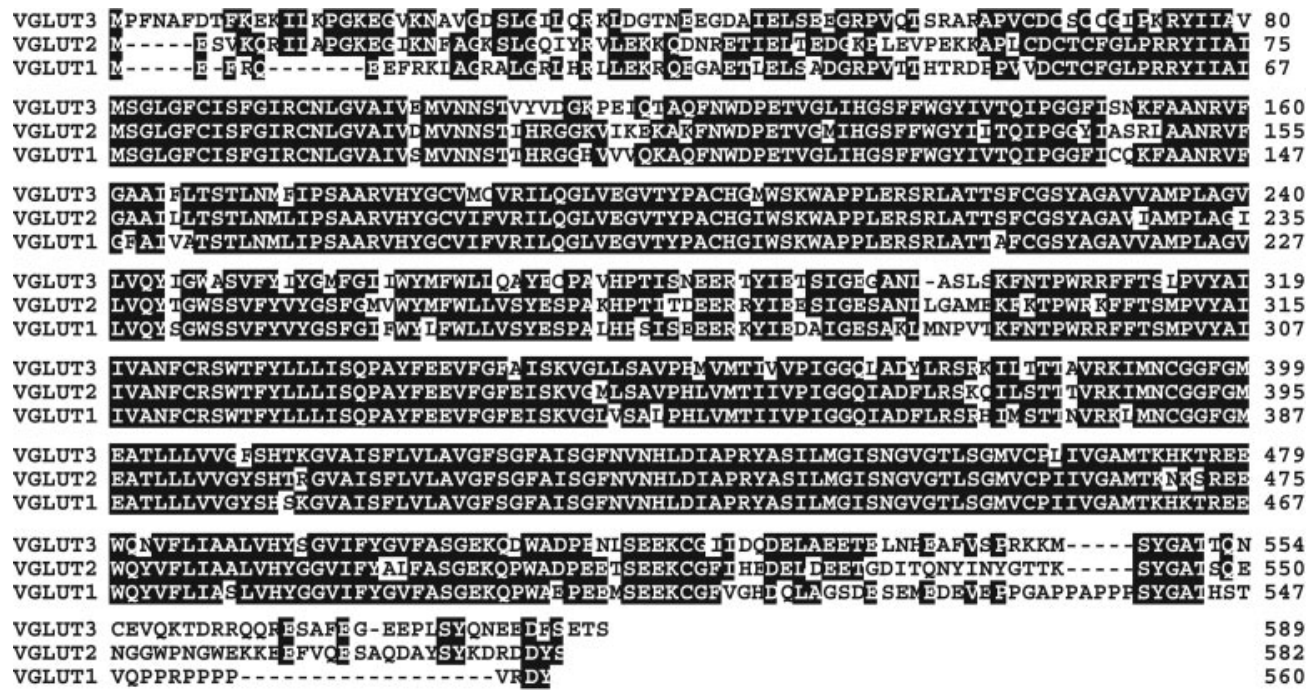

A

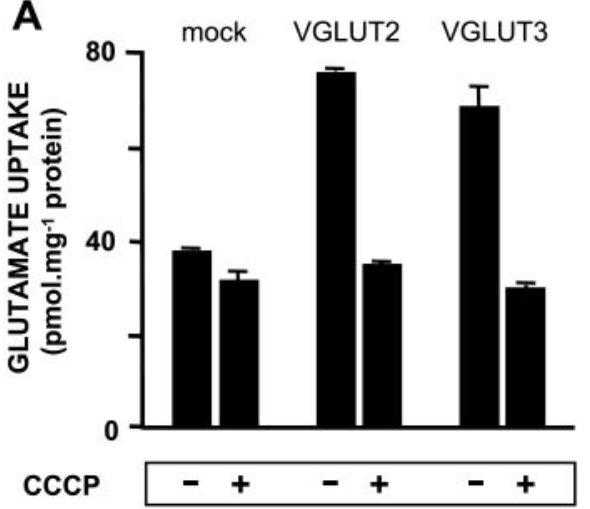

B

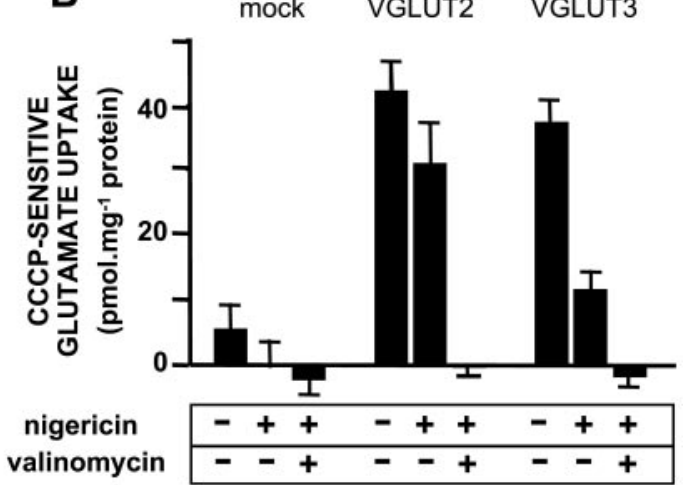

C

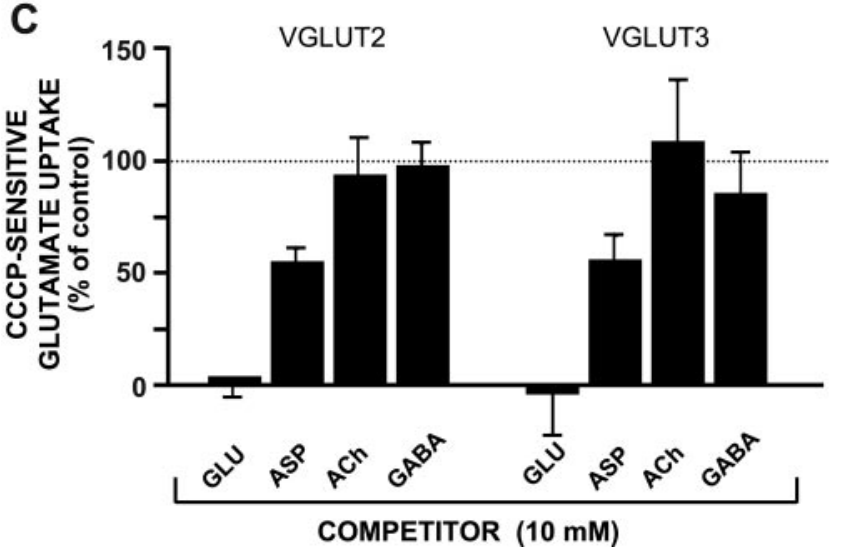

D

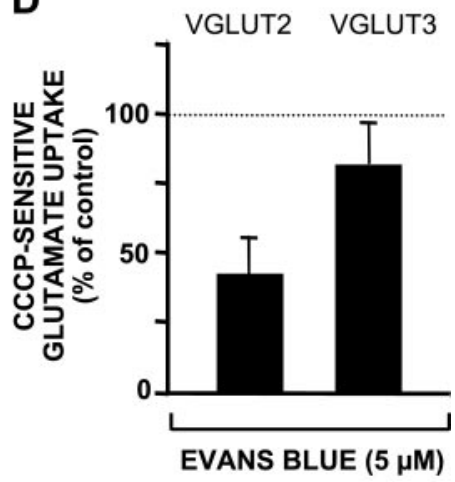

Figure 1. Alignment of VGLUT3, VGLUT2, and VGLUT1 amino acid sequences. The three proteins are highly conserved in their central portion. (Black boxing indicates identical residues.)
Figure 2. Functional characterization of VGLUT3. Membranes from BON cells stably expressing VGLUT3, VGLUT2, or an unrelated VIAAT (mock) were incubated at $30^{\circ} \mathrm{C}$ for 10 min with $40 \mu \mathrm{M}$ or $2 \mu \mathrm{Ci}$ of $\left[{ }^{3} \mathrm{H}\right] \mathrm{L}-$ glutamate and the following additives as stated: $50 \mu \mathrm{M}$ CCCP, $5 \mu \mathrm{M}$ nigericin, 20 $\mu \mathrm{M}$ valinomycin, and $5 \mu \mathrm{M}$ Evans Blue, as well as $10 \mathrm{~mm}(C)$ L-glutamate $(G L U)$, L-aspartate $(A S P)$, acetylcholine $(A c h)$, or GABA. Representative experiments are shown. The error bars represent the SEM of triplicate determinations. $A$, Expression of VGLUT2 or VGLUT3 induces a CCCP-sensitive uptake of glutamate. In $B-D$, only the specific (CCCP-sensitive) component of uptake is shown. In $C$ and $D$, the subtracted CCCP-resistant component was determined in the presence of an identical concentration of amino acid or Evans Blue. B, VGLUT3-mediated uptake is more sensitive to nigericin than VGLUT2. $C$, Both transporters prefer L-glutamate over other amino acids or transmitters. $D$, VGLUT3 is less sensitive to Evans Blue than VGLUT2. The dotted lines in $C$ and $D$ represent the control level (i.e., 100\%). 
A

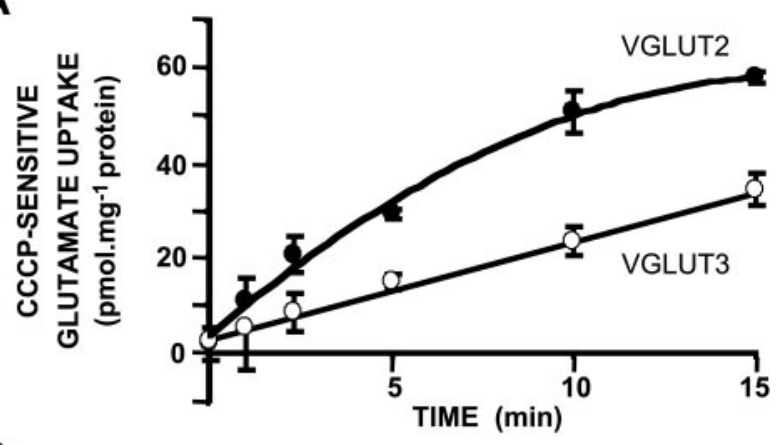

B
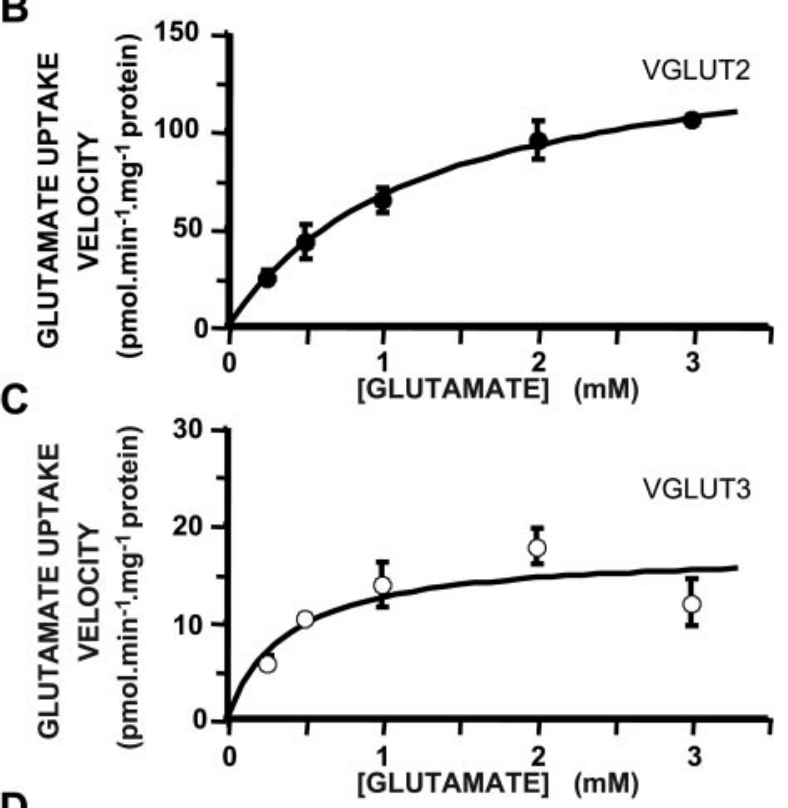

D

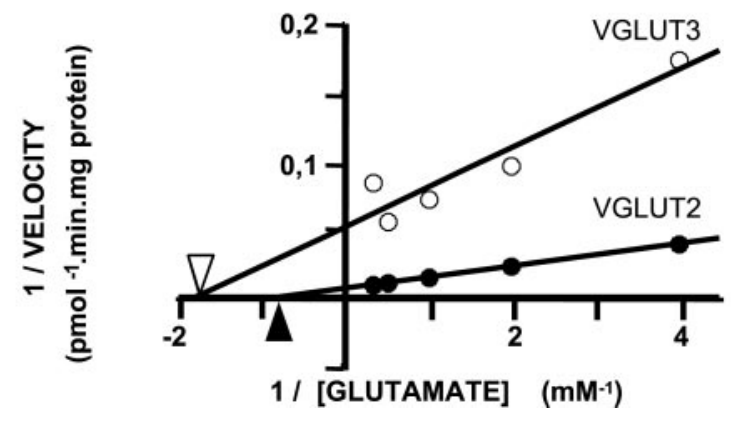

Figure 3. Saturation kinetics of VGLUT2 and VGLUT3. A, Time course of VGLUT2- and VGLUT3-mediated uptake at a $40 \mu \mathrm{M}\left[{ }^{3} \mathrm{H}\right]$ glutamate. $B$, The initial rate of uptake (at $3 \mathrm{~min}$ ) into VGLUT2-containing vesicles was determined with increasing concentrations of $\left[{ }^{3} \mathrm{H}\right]$ glutamate $(0.25-3 \mathrm{~mm})$. Specific uptake was determined at each glutamate concentration by subtracting the background uptake observed in the presence of $50 \mu \mathrm{M} \mathrm{CCCP.}$ $C$, Dependence of the initial rate of VGLUT3-mediated uptake (at $10 \mathrm{~min}$ ) on glutamate concentration was determined as in $B$. D, A LineweaverBurke plot of the data shown in $B$ and $C$ indicates $K_{\mathrm{M}}$ values of 1.27 and $0.56 \mathrm{~mm}$ and $V_{\max }$ values of 152 and $19 \mathrm{pmol} \cdot \mathrm{mg}^{-1}$ protein $\cdot \mathrm{min}^{-1}$ for VGLUT2 (closed circles) and VGLUT3 (open circles), respectively. Regression lines for VGLUT2 $(r=0.9993)$ and VGLUT3 $(r=0.8959)$ intersect the $1 / \mathrm{S}$ axis at distinct $-1 / K_{\mathrm{M}}$ values (closed and open arrowheads). The results in $B-D$ represent the average of three independent paired analyses of VGLUT2 and VGLUT3, each performed in triplicate. Error bars in $A-C$ correspond to SEM. membrane filter (Millipore, Bedford, MA), and three washes with $3 \mathrm{ml}$ of ice-cold $0.15 \mathrm{M} \mathrm{KCl}$. The radioactivity retained on the filters was measured by scintillation counting in Ready Protein ${ }^{+}$cocktail (Beckman, Fullerton, CA). Each uptake measurement was performed in triplicate and is expressed as mean \pm SEM. All experiments were independently performed three or more times on two independent BON-VGLUT3 clones. In each experiment, membranes from VGLUT3-expressing clones were compared with those of VGLUT2 or mock (VIAAT-expressing) transfectants.

$R T$-PCR. RT-PCR detection of VGLUT3 mRNA in body organs and in different brain areas was performed as described previously (Antunes Bras et al., 1998) with the following primers: 5'-ACCCGGGAAGAATGGCAGAATCTG-3' and 5'-ATGGGAAAAGCAATGGGTGTGGAG-3'. RT-PCR generated product with glyceraldehyde-3-phosphate dehydrogenase (G3PDH)-specific probes (Clontech, Palo Alto, CA) was used to normalize the RNA level in tissue sample extracts.

In situ hybridization. Regional in situ hybridization was performed with a mix of six antisense oligonucleotides (5'-GTAAGATCCCCAGCGAATCTCCCACGGCAT-3', 5' -CAATAGGAGAGGCACCTCAGAGCCCTTAGC-3', 5'-ACTCAGCTCAATGGCATCTCCCTCCTCGTT-3', 5'-TTCATTCTGGTAGGATAATGGCTCCTCCCC-3', 5'-GGATTCTCTCTGTTGTCTCCGATCCGTCTT-3', and 5'-GACCTCACAATTCTGGGTGGTGGCTCCATA-3') as described previously (Davis et al., 2000; Herzog et al., 2001). In brief, oligonucleotides were labeled with $\left.{ }^{35} \mathrm{~S}\right] \mathrm{dATP}$, using terminal transferase (Amersham Biosciences), to a specific activity of $5 \times 10^{-8} \mathrm{dpm} / \mu \mathrm{g}$. Sections were covered with $100 \mu \mathrm{l}$ of a solution containing 50\% hybridization solution (Amersham Biosciences), 40\% deionized formamide (Merck Eurolab, Strasbourg, France), $500 \mu \mathrm{g} / \mathrm{ml}$ poly(A) (Roche, Meylan, France), $100 \mathrm{~mm}$ 4-dithiothreitol, and 3-5 $\times 10^{-5} \mathrm{dpm}$ of each labeled oligonucleotide. The samples were incubated overnight at $42^{\circ} \mathrm{C}$, washed, and exposed to $\mathrm{x}$-ray films (Biomax; Eastman Kodak, Rochester, NY) for $21 \mathrm{~d}$.

The plasmids used to synthesize the cRNA probes for cold in situ hybridization were obtained by PCR amplification with the following primer couples: 5'-ACTGTTACCAAGATGCCC-3' and 5'-ATGAGCACGAACCATTCC $-3^{\prime}$ for the rat serotonin transporter (SERT) and $5^{\prime}$ AAAACAGGACTGGGCTGATCC- $3^{\prime}$ and $5^{\prime}$-GAGACCAAGATCCATACGCCC-3' for VGLUT3. The choline acetyltransferase (ChAT) plasmid used for cold in situ hybridization was a generous gift from $\mathrm{S}$. Berrard (CNRS UMR7091, Paris, France). Double cold in situ hybridization was performed with antisense riboprobes labeled with either fluorescein-UTP (for ChAT or SERT) or digoxygenin (DIG)-UTP (for VGLUT3) (Herzog et al., 2001). Colorimetric revelations were obtained with 5-bromo-4-chloro-3-indolyl phosphate (Roche) and either nitroblue tetrazolium (Roche) for VGLUT3 or 2-[4-iodophenyl]-3-[4-nitrophenyl]-5phenyl-tetrazolium chloride (Roche) for ChAT and SERT, to obtain the blue and red staining, respectively.

Antiserum. Two anti-VGLUT3 antisera, named P45-1 and P45-3, were obtained by immunizing rabbits (Agro-Bio, Villeny, France) against the following peptides, coupled via their cysteine residues to keyhole limpet hemocyanin: CDSLGILQRKLDGTNEEGD (Asp $\left.{ }^{27}-\mathrm{Asp}^{44}\right)$, and CETELNHEAFVSPRKKM $\left(\mathrm{Glu}^{531}-\mathrm{Met}^{547}\right)$, respectively. Both antisera were decomplemented by heating $30 \mathrm{~min}$ at $56^{\circ} \mathrm{C}$, dialyzed, and stored in the presence of $50 \%$ glycerol at $-20^{\circ} \mathrm{C}$. For immunological detection, the antisera were affinity purified on peptides linked to Affigel-15 (Bio-Rad, Richmond, CA).

Immunoautoradiography. Immunoautoradiography was performed as described previously (Herzog et al., 2001). In brief, adult male Sprague Dawley rats were anesthetized and perfused via the ascending aorta with $200 \mathrm{ml}$ of $0.9 \% \mathrm{NaCl}$ containing sodium nitrite $(1 \mathrm{gm} / 1)$. Brains were dissected and frozen in isopentane at $-30^{\circ} \mathrm{C}$. Horizontal or coronal rat brain sections were fixed with $4 \%$ paraformaldehyde and washed with PBS containing 3\% bovine serum albumin (BSA), $1 \%$ goat serum, and 1 mM NaI (buffer A). Sections were incubated with buffer supplemented with affinity-purified VGLUT3 antiserum (1:5000 dilution) and then with [ $\left.{ }^{125} \mathrm{I}\right] \mathrm{IgG}(0.25 \mu \mathrm{Ci} / \mathrm{ml}$; Amersham). Sections were apposed to $\mathrm{x}$-ray films (Biomax; Kodak) for $5 \mathrm{~d}$.

Immunohistochemistry. Immunocytochemistry was performed as described previously (Herzog et al., 2001). Adult male Sprague Dawley rats were anesthetized and perfused intracardially with $300 \mathrm{ml}$ of $120 \mathrm{~mm}$ sodium phosphate buffer ( $\mathrm{PB}, \mathrm{pH}$ 7.4) supplemented with $4 \%$ paraformaldehyde. The brains were dissected, postfixed by immersion in the same fixative, and cryoprotected in PB containing $30 \%$ sucrose. Coronal sections were taken at $-20^{\circ} \mathrm{C}$ and mounted on glass slides. Sections were washed with PB containing gelatin $(2 \mathrm{gm} / \mathrm{l})$ and Triton X-100 $(0.25 \%)$ and incubated with VGLUT3 antiserum in the presence or absence of 
A

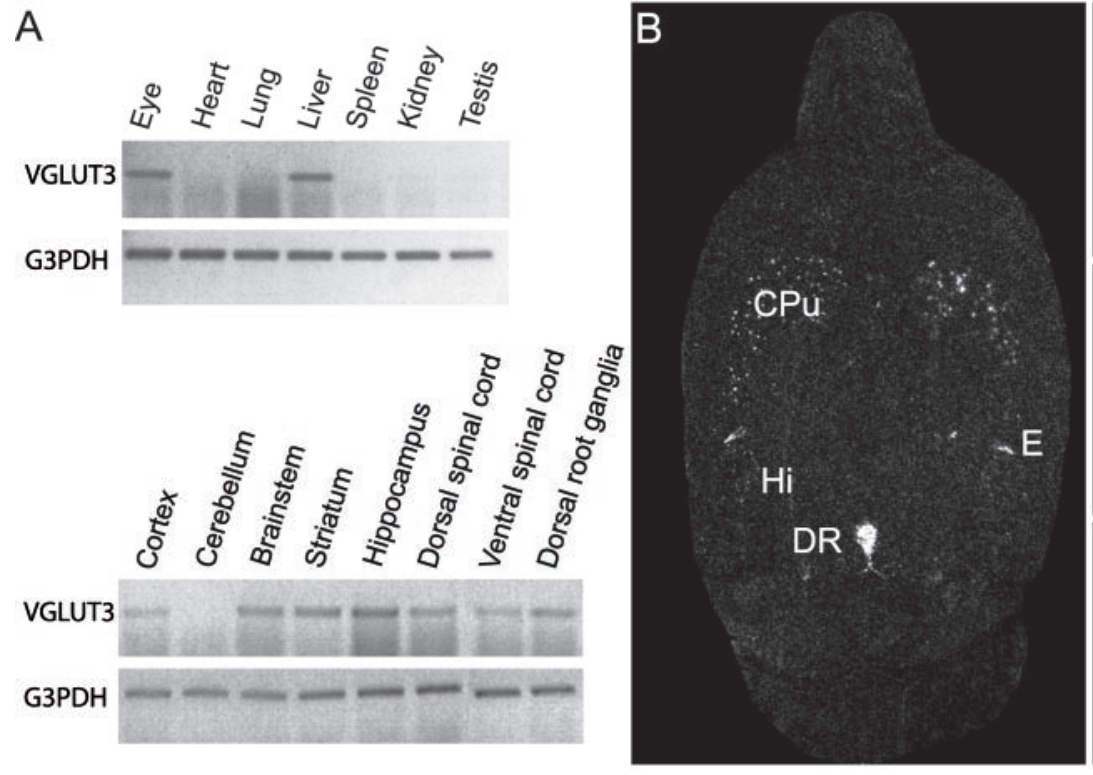

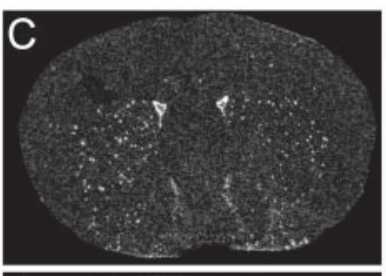

$F$
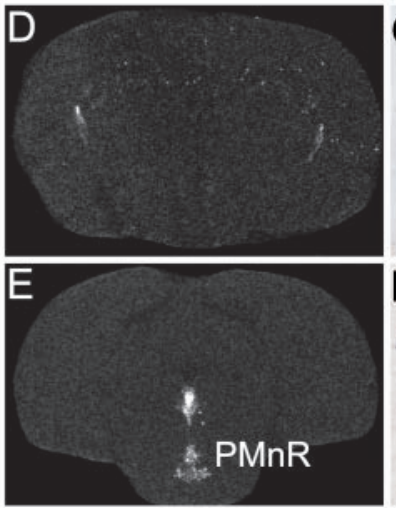

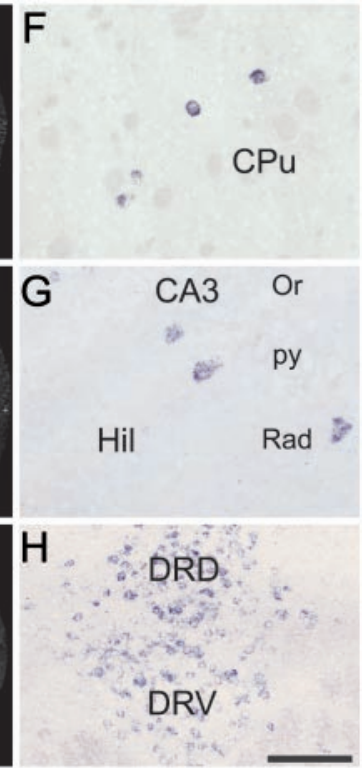

Figure 4. Regional distribution of VGLUT3 mRNA. A, RT-PCR detection of VGLUT3 mRNA in body organs and in different brain areas. G3PDH-specific primers (Clontech) were used to normalize the RNA level in tissue sample extracts. $B-H$, In situ hybridization analysis of VGLUT3 transcript distribution in the rat brain. Horizontal $(B)$ or coronal $(C-H)$ brain sections were hybridized with antisense ${ }^{35} \mathrm{~S}$-labeled oligonucleotides $(B-E)$ or DIG-UTP-labeled cRNA probes $(F-H)$. $C A 3$, CA3 field of the hippocampus; $C P u$, caudate-putamen; $D R$, dorsal raphe; $D R D$, dorsal part of the dorsal raphe nucleus; $D R V$, ventral part of the dorsal raphe nucleus; $E$, ependymal cells; $H i$, hippocampus; Hil, hilus of the dentate gyrus; Or, oriens layer of the hippocampus; $P M n R$, paramedian raphe nuclei; $p y$, pyramidal layer of the hippocampus; Rad, radiatum layer of the hippocampus. Scale bars: $F, H, 200 \mu \mathrm{m} ; G, 100 \mu \mathrm{m}$.

guinea pig anti-vesicular acetylcholine transporter (VAChT) antiserum (Chemicon, Temecula, CA). VGLUT3 alone was detected with antirabbit IgG coupled to Alexa Fluor 568 dye (Molecular Probes, Eugene, OR). VGLUT3 and VAChT were codetected by immunofluorescence, using goat anti-rabbit IgG coupled to CY3 and goat anti-guinea-pig coupled to FITC, respectively, as secondary antibodies. The sections were observed using a conventional (Axioskop 2 Plus; Zeiss, Thornwood, NY) or a confocal laser scanning (LSM 410; Zeiss) microscope.

VGLUT3 was visualized at the electron-microscopic level using the pre-embedding immunogold method with silver intensification (Bernard et al., 1999). Briefly, the sections were incubated in goat anti-rabbit IgGs conjugated to gold particles (0.8 nm diameter; $1: 100$ in PBS/acetylated BSA (BSA-C); Aurion, Wageningen, The Netherlands) for $2 \mathrm{hr}$ in PBS/BSA-C. The sections were then washed $(3 \times$ PBS $)$ and postfixed in $1 \%$ glutaraldehyde in PBS for $10 \mathrm{~min}$. After washing $(2 \times \mathrm{PBS} ; 2 \times$ sodium acetate buffer, $0.1 \mathrm{M}, \mathrm{pH} 7.0$ ), the diameter of the gold immunoparticles was increased using a silver enhancement kit (HQ silver; Nanoprobes, Yaphank, NY) for 5 min at room temperature in the dark. After treatment with $1 \%$ osmium, dehydration, and embedding in resin, ultrathin sections were cut, stained with lead citrate, and examined using a Philips (Eindhoven, The Netherlands) CM10 electron microscope or a Philips Tecnai 20. VGLUT3 and VAChT were codetected at the electronmicroscopic level by pre-embedding immunogold and immunoperoxidase, respectively. Briefly, the sections were incubated in a mixture of goat anti-rabbit IgGs conjugated to gold particles ( $0.8 \mathrm{~nm}$ diameter; 1:100 in PBS/BSA-C; Aurion) and goat anti-guinea pig coupled to biotin (1:200) for $2 \mathrm{hr}$ in PBS/BSA-C. The sections were then washed ( $3 \times$ PBS) and postfixed in $1 \%$ glutaraldehyde in PBS for $10 \mathrm{~min}$. After washing $(2 \times$ PBS; $2 \times$ sodium acetate buffer, $0.1 \mathrm{M}, \mathrm{pH} 7.0)$, the diameter of the gold immunoparticles was increased using a silver enhancement kit (HQ silver; Nanoprobes) for $5 \mathrm{~min}$ at room temperature in the dark. The sections were finally washed in acetate buffer and in PBS and incubated in an avidin-biotin-peroxidase complex (1:100; Vector Laboratories, Burlingame, CA) for $1.5 \mathrm{hr}$ at room temperature. After washing $(2 \times$ PBS; $1 \times$ Tris buffer, $0.05 \mathrm{M}, \mathrm{pH}$ 7.6), the immunoreactive sites for VAChT were revealed using DAB. The sections were treated with $1 \%$ osmium, dehydrated, and embedded in resin. Ultrathin sections were cut, stained with lead citrate, and examined in the electron microscope.

\section{RESULTS}

\section{Cloning and functional characterization of VGLUT3}

A rat hippocampus cDNA library was screened with a combination of VGLUT1 and VGLUT2 probes (Herzog et al., 2001). Of $6 \times 10^{-5}$ plated phages, 55 positive clones were isolated and analyzed. Thirty-seven were identical to VGLUT1 and four were identical to VGLUT2. One cDNA, designated P45, encodes a 588 aa protein (calculated $M_{\mathrm{r}} \approx 64,700$ ). $\mathrm{P} 45$ is highly homologous to VGLUT1 and VGLUT2 ( $>70 \%$ aa identity). Most of the divergences between P45, VGLUT1, and VGLUT2 sequences are concentrated in the $\mathrm{N}$ and $\mathrm{C}$ termini (Fig. 1).

To analyze whether P45 represents a novel glutamate transporter, its cDNA was stably expressed in the serotoninergic endocrine cell line BON (Evers et al., 1991). Intracellular membrane vesicles were purified from two independent positive clones (see Fig. $5 C$ ) and analyzed for their capacity to take up $\left[{ }^{3} \mathrm{H}\right]$ glutamate in the presence of ATP. In each experiment, these vesicles were compared with membranes derived from mock- and VGLUT2-transfected cells. P45-containing vesicles, as VGLUT2containing membranes, accumulated approximately twice as much $\left[{ }^{3} \mathrm{H}\right]$ glutamate as mock vesicles (Fig. $2 A$ ). Inhibition of this transport by the $\mathrm{H}^{+}$ionophore CCCP indicated that the increased uptake observed in P45- and VGLUT2-containing membranes was attributable to an $\mathrm{H}^{+}$-driven transport, which was $3.07 \pm 0.17$ and $4.42 \pm 0.28$ times higher than in mock vesicles, respectively (means $\pm \mathrm{SEM} ; n=26$ ). For clarity, only the CCCP-sensitive component of uptake is considered hereafter. Because the P45-mediated accumulation of $\left[{ }^{3} \mathrm{H}\right]$ glutamate remained linear for 15 min (Fig. $3 A$ ), we used a constant duration of $10 \mathrm{~min}$ throughout this study.

The bioenergetic properties of P45- and VGLUT2-mediated 

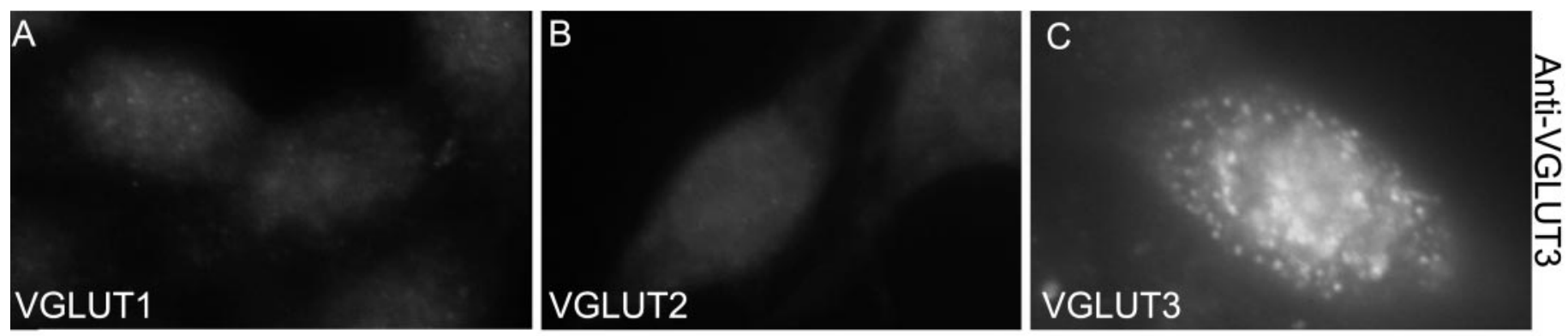

D

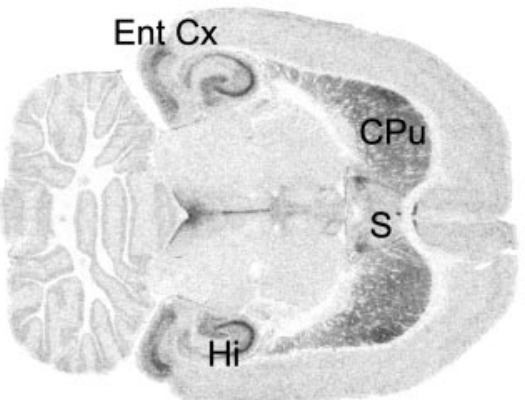

$\mathrm{E}$

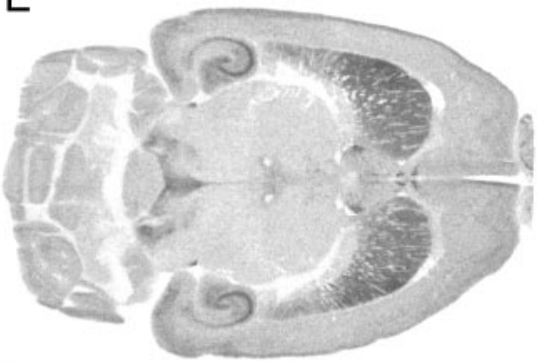

$\mathrm{H}$

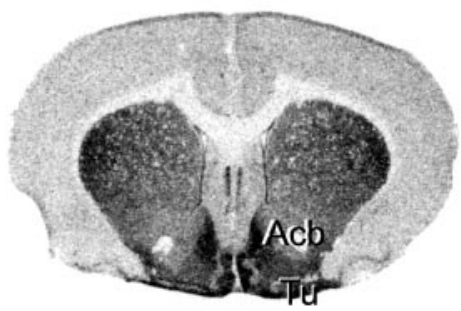

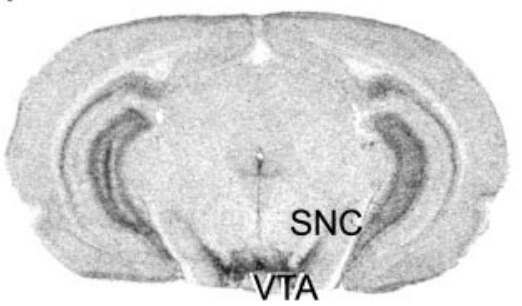

$\mathrm{F}$

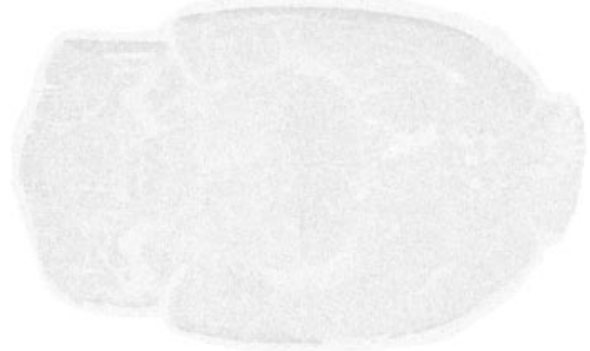

I

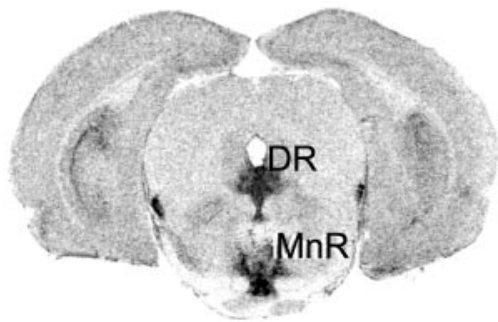

Figure 5. Antiserum specificity and regional distribution of VGLUT3. Two polyclonal antisera directed against distinct peptides were generated. $A-C$, Immunofluorescence detection of VGLUT3 in BON stable transfectants (white puncta) with the P45-1 antiserum at a dilution of 1:5000. The serum recognizes VGLUT3 but not VGLUT1 or VGLUT2 in BON stable transfectants. The same result was obtained with the P45-3 antiserum (data not shown). $D-I$, Localization of VGLUT3 protein by immunoautoradiography. In $D$ and $G-I$ the P45-3 antiserum was used. In $E$ and $F$, the P45-1 antiserum was used in the presence $(F)$ or absence $(E)$ of its cognate peptide $(0.1 \mathrm{mg} / \mathrm{ml})$. $A c b$, accumbens; $C P u$, caudate-putamen; $D R$, dorsal raphe; Ent $C x$, entorhinal cortex; $H i$, hippocampus; $M n R$, median raphe nucleus; $S$, septum; $S N C$, substantia nigra pars compacta; $T u$, olfactory tubercles; $V T A$, ventral tegmental area. Scale bars: $J, L, N, 200 \mu \mathrm{m} ; K, M, O, 50 \mu \mathrm{m}$.

processes were compared by adding nigericin, an ionophore that collapses transmembrane $\mathrm{pH}$ gradients by exchanging $\mathrm{H}^{+}$for $\mathrm{K}^{+}$. As illustrated in Figure $2 B$, although nigericin moderately inhibited VGLUT2 by $31.7 \pm 2.3 \%$, as reported previously (Bai et al., 2001; Fremeau et al., 2001; Herzog et al., 2001; Varoqui et al., 2002), a stronger inhibition (70.4 $\pm 6.1 \%)$ was reproducibly observed for P45 (paired $t$ test: $p<0.001 ; n=6$ ). Therefore, the $\mathrm{P} 45$-mediated process is more dependent on the chemical component of the $\mathrm{H}^{+}$electrochemical gradient, suggesting that it might translocate more $\mathrm{H}^{+}$than VGLUT2. Both uptakes were abolished by a further addition of valinomycin, a $\mathrm{K}^{+}$ionophore that disrupts the remaining electrical component by exporting the $\mathrm{K}^{+}$ions accumulated by nigericin (Fig. $2 B$ ).

The substrate selectivity of P45 was assessed by applying unlabeled amino acids and/or neurotransmitters simultaneously with $\left[{ }^{3} \mathrm{H}\right]$ glutamate (Fig. $\left.2 \mathrm{C}\right)$. L-glutamate $(10 \mathrm{~mm})$ inhibited the P45-mediated transport by $92.1 \pm 4.1 \%(n=6)$, whereas L-aspartate only partially inhibited uptake $(56.5 \pm 4.2 \%$ inhibition; $n=6)$. Acetylcholine $(n=5)$ or $\operatorname{GABA}(n=4)$ at the same concentration (Fig. $2 C$ ) or serotonin at $0.5 \mathrm{~mm}$ (i.e., approximately two orders of magnitude over its cytosolic concentration in serotoninergic cells) (data not shown) had no effect. The selectivity for L-glutamate over L-aspartate was confirmed by the fact that we could not detect any CCCP-sensitive accumulation of $\left[{ }^{3} \mathrm{H}\right]$ aspartate induced by $\mathrm{P} 45$ (data not shown). Similar results were obtained for VGLUT2 (Fig. 2C), as reported previously (Bai et al., 2001; Fremeau et al., 2001; Herzog et al., 2001; Takamori et al., 2001; Varoqui et al., 2002). In contrast, Evans Blue (5 $\mu \mathrm{M})$, a compound that competitively inhibits glutamate uptake into synaptic vesicles (Roseth et al., 1995), discriminated the P45- and VGLUT2-mediated processes (Fig. 2D), because inhibitions of $16.5 \pm 5.6$ and $55.9 \pm 3.4 \%$ were observed, respectively (paired $t$ test: $p<0.005 ; n=4)$. To characterize further the interaction of $\mathrm{P} 45$ with glutamate, increasing concentrations of $\left[{ }^{3} \mathrm{H}\right]$ glutamate were tested. As illustrated in Figure $3 C, D$, the P45-mediated uptake followed Michaelis-Menten kinetics, with mean $V_{\max }$ and $K_{\mathrm{M}}$ values of $20.3 \pm 4.8 \mathrm{pmol} \cdot \mathrm{mg}^{-1} \operatorname{protein} \cdot \min ^{-1}$ and $0.52 \pm$ $0.21 \mathrm{~mm}(n=3)$. A similar analysis of VGLUT2 kinetics performed in parallel yielded a much higher $V_{\max }(168 \pm 20$ $\mathrm{pmol} \cdot \mathrm{mg}^{-1}$ protein $\left.\cdot \mathrm{min}^{-1}\right)$ and a slightly higher $K_{\mathrm{M}}(1.46 \pm$ $0.35 \mathrm{~mm}$ ) (Fig. $3 B, D$ ). The slower $V_{\max }$ of $\mathrm{P} 45$, observed with both BON transfectants, may originate from a lower expression of the protein and/or a slower turnover of the transport cycle. To confirm the difference in substrate affinity, additional competition experiments were performed with submillimolar concentrations of unlabeled amino acids (data not shown). Indeed, we found that 
a $0.5 \mathrm{~mm}$ concentration of glutamate inhibited P45 more efficiently (by $80.8 \pm 3.6 \% ; n=5$ ) than VGLUT2 $(46.9 \pm 3.0 \%)$. This difference was highly significant (paired $t$ test: $p<0.005 ; n=$ 5). In conclusion, P45 transports glutamate with characteristics very similar, but not identical, to those displayed by VGLUT1 and VGLUT2. Consequently, it was renamed VGLUT3.

\section{Anatomic distribution of VGLUT3}

VGLUT3 mRNA distribution in body organs was analyzed by reverse transcription and amplification by PCR (Fig. 4A). A 398 $\mathrm{kb}$ amplification fragment was found in the brain, eyes, and liver (Fig. $4 A$ ). In the brain, VGLUT3 was detected in all inspected areas except the cerebellum (Fig. $4 A$ ).

This finding was confirmed and extended by in situ hybridization analysis, which revealed a very discrete pattern of expression (Fig. 4B-H). No signal is detected in the cerebellum and thalamus. High VGLUT3 mRNA expression is observed in the dorsal and medial raphe nuclei, caudate-putamen, and accumbens (Fig. $4 B, C, E$ ), whereas low levels are found in the hippocampus (Fig. $4 B, D)$ and habenula (data not shown). A strong signal was also observed in ependymal cells (Fig. 4B,D). At the cellular level, the labeling was concentrated over large, scattered neurons in the striatum (Fig. 4F), hippocampus (Fig. 4G), and cerebral cortex (data not shown). A high density of positive neurons was observed in the dorsal and ventral parts of the raphe dorsalis nucleus (Fig. $4 H$ ). Lower amounts of VGLUT3 mRNA are also present in the lateral parts of the dorsal raphe (Fig. $4 H$ ).

Thus, in contrast to VGLUT1 and VGLUT2 (Ni et al., 1994, 1995; Hisano et al., 2000; Fremeau et al., 2001; Herzog et al., 2001), only a few neurons of the brain express VGLUT3.

To examine the localization of the protein, two independent polyclonal antiserums were raised by immunizing rabbits against two different peptides of the VGLUT3 $\mathrm{N}$ and $\mathrm{C}$ terminus. These two anti-peptide antiserums, named $\mathrm{P} 45-1$ and $\mathrm{P} 45-3$, gave very similar, if not identical, results in all tested conditions. Both antiserums were able to detect a strong intracellular and punctiform signal on BON cells permanently transfected with pcDNA3VGLUT3, but not with plasmids coding for VGLUT1 or VGLUT2 (as shown in Fig. $5 A-C$ with P45-3; data not shown for $\mathrm{P} 45-1)$. On rat brain sections, the same discrete immunoautoradiographic labeling pattern was observed with the two antiserums (Fig. 5D,E). Finally, the immunolabeling of brain sections was not detected with a preimmune serum (data not shown) or antiserums saturated with their cognate peptides (Fig. $5 F$; data not shown). Together, these experiments demonstrate the specificity of the antiserums.

In contrast to its transcript, the VGLUT3 protein was relatively broadly distributed in the gray matter of the CNS (Fig. $5 D-I$ ). No signal was detected in the white matter. A strong labeling was observed in the caudate-putamen, accumbens (shell $>$ core), olfactory tubercles, hippocampus (pyramidal and granular cell layers), ventral tegmental area, substantia nigra (pars compacta), and raphe nuclei (Fig. 5G-I). Thus, VGLUT3 mRNA (Fig. 4) and protein (Fig. 5) are present in the same brain regions, but their distribution patterns are not similar. These differences imply that the protein VGLUT3 is not addressed exclusively to cell soma. Furthermore, the regional colocalization of VGLUT3 transcripts and protein suggests that VGLUT3 is expressed by striatal and hippocampal interneurons. However, VGLUT3 is also abundant in regions in which its mRNA is absent, such as the substantia nigra (pars compacta) and ventral tegmental area (Fig. 5H). The partial mismatch between the locations of the transcript and the
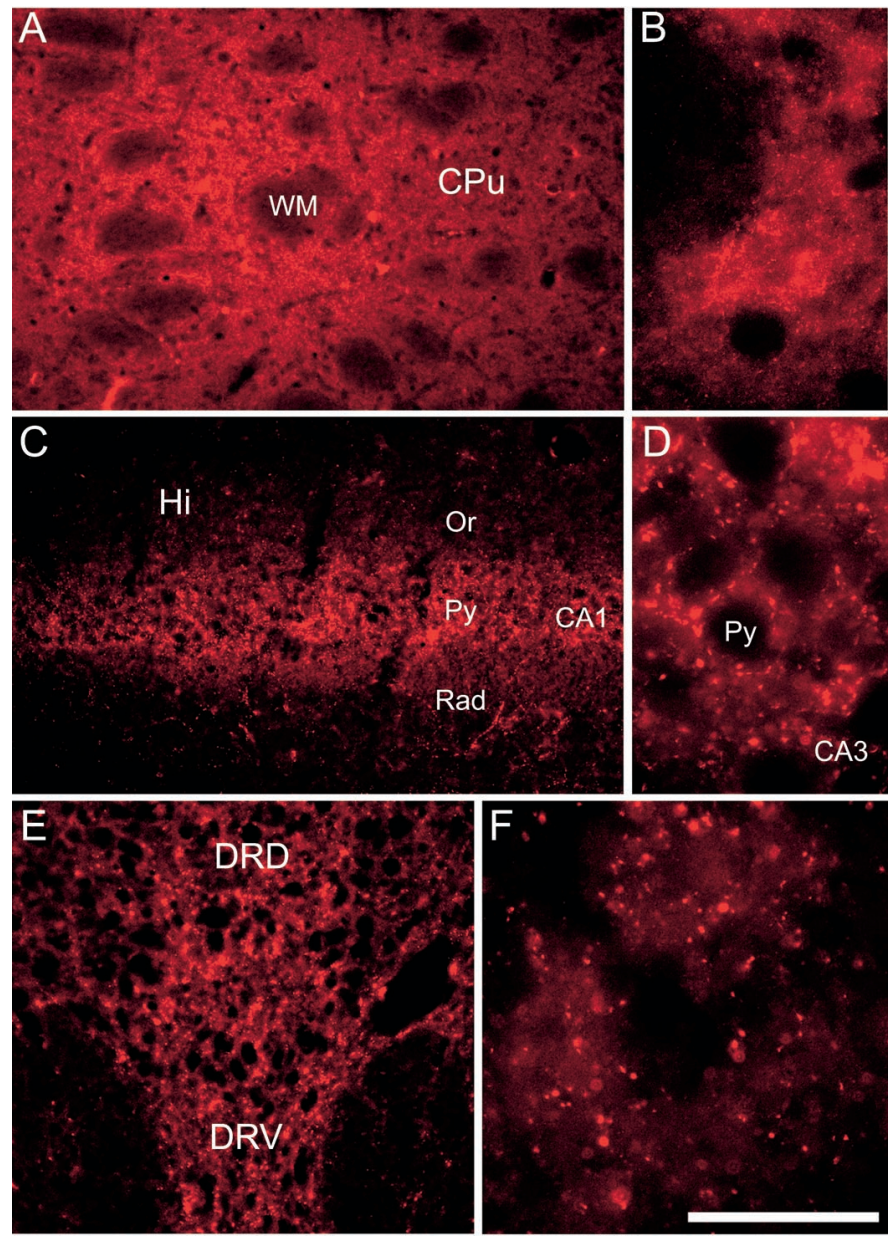

Figure 6. Immunofluorescent detection of VGLUT3 in the brain. VGLUT3 immunoreactivity appears as red puncta in the caudateputamen $(A, B)$, hippocampus $(C, D)$, and dorsal raphe $(E, F)$. CA1, CA1 field of the hippocampus; $C A 3, \mathrm{CA} 3$ field of the hippocampus; $C P u$, caudate-putamen; $D R D$, dorsal part of the dorsal raphe nucleus; $D R V$, ventral part of the dorsal raphe nucleus; $\mathrm{Hi}$, hippocampus; $\mathrm{Or}$, oriens layer of the hippocampus; $p y$, pyramidal layer of the hippocampus; $R a d$, radiatum layer of the hippocampus; $W M$, white matter. Scale bars: $A, C, E, 200$ $\mu \mathrm{m} ; B, D, F, 50 \mu \mathrm{m}$.

protein suggests that VGLUT3 is also expressed at the terminals of principal neurons.

At the light microscopic level, the caudate-putamen, hippocampus, and raphe nuclei exhibited an intense punctiform labeling of their neuropil (Fig. 6). VGLUT3 is evenly distributed in the entire striatal neuropil but not in fiber track patches (Fig. 6A,B). At higher magnification, this labeling appears as multiple small puncta (Fig. 6B). In the hippocampus, VGLUT3-positive neurons, although very scattered (Fig. $4 B, D, G$ ), produce a dense network surrounding the soma of pyramidal and granular cells (Fig. 6C,D). VGLUT3 puncta are widespread in the raphe dorsalis (Fig. 6E,F). In the hippocampal formation and raphe nucleus, the puncta have a somewhat larger size than in the striatum (Fig. 6B,D,F).

\section{Localization of VGLUT3 over synaptic vesicle clusters}

At the electron-microscopic level, the above-described puncta were found to correspond to nerve endings in the striatum, hippocampus, and raphe nucleus (Fig. $7 A-E$ ), as well as all other inspected brain areas (data not shown). The terminals were 
Figure 7. VGLUT3 is localized in nerve endings in the striatum $(A)$, hippocampus $(B, C)$, and dorsal raphe $(D, E)$. In all three areas, immunoparticles for VGLUT3 are localized in terminals $(t)$. In the striatum, dorsal raphe, and hippocampus $(C)$, the labeled terminals are in close apposition with dendrites $(d)$. In the hippocampus and dorsal raphe, the immunoreactive terminal makes a symmetrical synapse (arrowheads) with a pyramidal cell (Pyr Cell) in B and D; one terminal makes an asymmetrical synapse (arrow) with a dendrite $(d)$ in $C$ and $E$. Scale bar, $250 \mathrm{~nm}$.
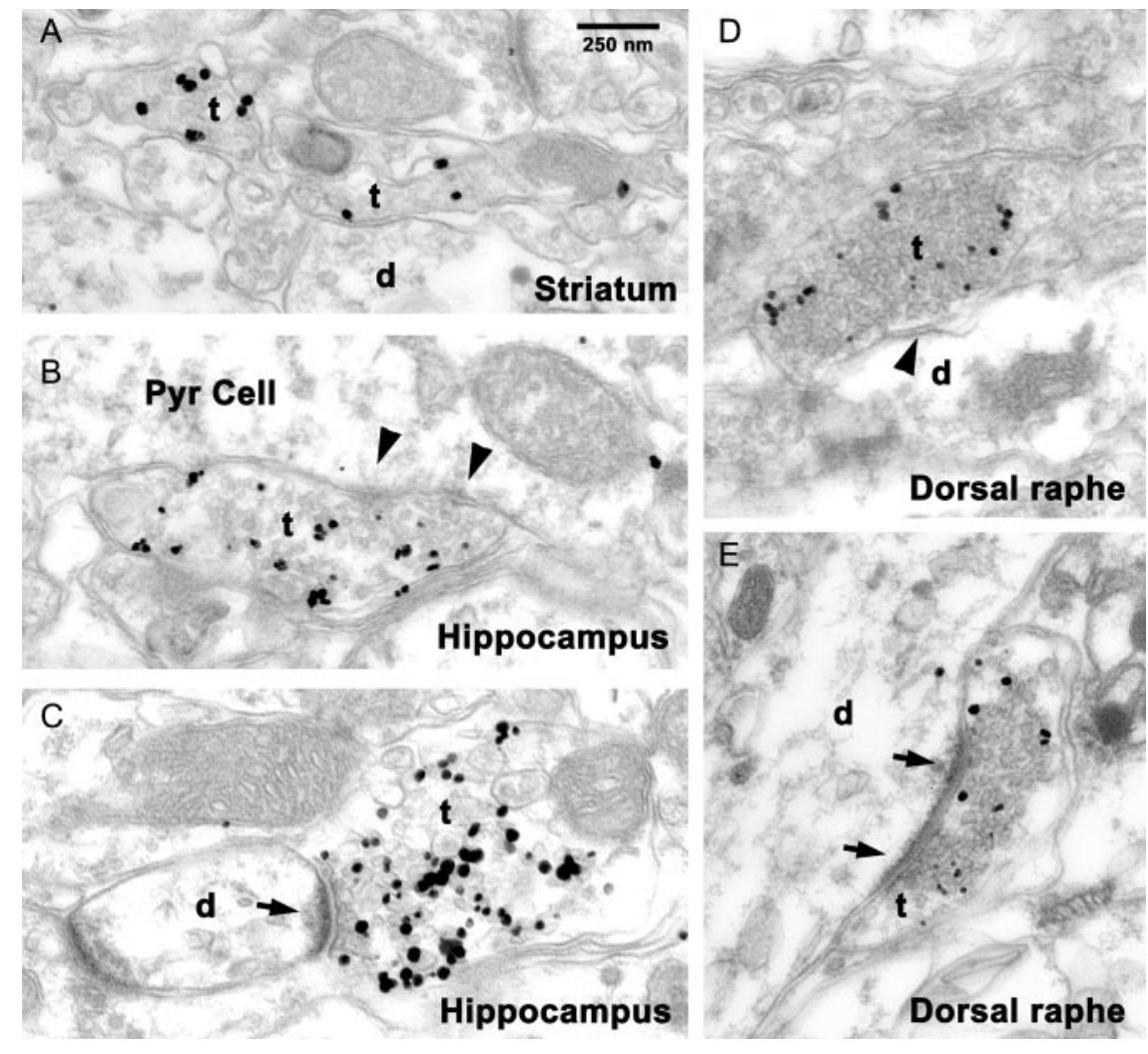

identified by the presence of synaptic vesicles. Immunoparticles for VGLUT3 accumulated over vesicle clusters in terminals making classical asymmetrical synapses in the hippocampus (Fig. $7 C$, arrows) and raphe nucleus (Fig. $7 E$, arrows). However, in both structures a large number of VGLUT3-positive terminals are also forming symmetrical synapses (Fig. 7B,D, arrowheads). The VGLUT3-immunoreactive terminals made close appositions or synaptic contacts with different parts of the neurons. In the CA3 field of the hippocampus, labeled terminals made symmetrical and asymmetrical contacts with dendrites and symmetrical contacts with perikarya of pyramidal neurons. In the dorsal raphe, VGLUT3-positive terminals made symmetrical and asymmetrical synapses with dendrites. In the striatum, VGLUT3 immunolabeled terminals made close appositions and symmetrical contacts with dendrites.

Because this finding does not fit with the conventional view that glutamate synapses make asymmetrical contacts, we investigated the nature of the VGLUT3-positive neurons in more detail.

\section{VGLUT3 is expressed in cholinergic and serotoninergic neurons}

The sparse VGLUT3-positive giant cells in the caudate-putamen nucleus are reminiscent of cholinergic neurons, as detected with a probe for the ChAT transcript (Fig. 8A,C). We thus compared the expression of the VGLUT3 and ChAT mRNAs, using in situ hybridization with double colorimetric detection. As illustrated in Figure $8 B, D$, we found in the caudate-putamen that every ChATpositive neuron (detected by a red precipitate) also expressed VGLUT3 (labeled in blue).

Laser confocal microscopy was then used to investigate the potential colocalization of VGLUT3 with another specific marker of cholinergic nerve endings, the VAChT, at the protein level (Gilmor et al., 1996; Roghani et al., 1996; Weihe et al., 1996). In the hippocampus (Fig. $8 F$ ) or the septum (data not shown), VGLUT3 (red fluorescence) and VAChT (in green) are clearly present in two distinct sets of nerve terminals. In contrast, in the striatum, numerous thin varicose fibers are labeled in yellow fluorescence, very few are in red, and green terminals are absent (Fig. $8 E$ ). This experiment suggests that the vast majority of striatal cholinergic terminals contain VGLUT3. Some noncholinergic VGLUT3-positive nerve endings are observed that may originate from brain regions sending projections to the striatum.

In the experiment depicted in Figure $8 G$, VAChT is detected by immunoperoxidase and VGLUT3 is detected by immunogold at the ultrastructural level. As shown in Figure $8 G$, two small terminals, containing both the peroxidase precipitate and immunogold particles, are VAChT- as well as VGLUT3-positive, respectively. Thus, the colocalization of VGLUT3 and VAChT in striatal nerve terminals is also confirmed at the electronmicroscopic level.

Together, these results clearly show that almost all cholinergic striatal interneurons express VGLUT3 at their nerve endings.

As shown by in situ hybridization and immunoautoradiography, VGLUT3 is also strongly expressed in the serotoninergic raphe nuclei (Figs. $4 E, H, 9 A, B$ ). We thus analyzed by double in situ hybridization whether VGLUT3 is present in serotoninergic neurons. In Figure 9, VGLUT3-positive neurons are in blue and serotoninergic neurons, which labeled the plasma membrane SERT transcript, are detected by a red precipitate. As demonstrated by this experiment, all of the SERT-positive neurons also expressed VGLUT3 in the dorsal and median raphe (Fig. 9A,B). A red/blue neuron is shown at a higher magnification on Figure $9 C$. Interestingly, numerous neurons from the dorsal raphe are expressing VGLUT3 but not SERT. Accordingly, nonserotoninergic neurons from the raphe dorsalis, which are VGLUT3- 


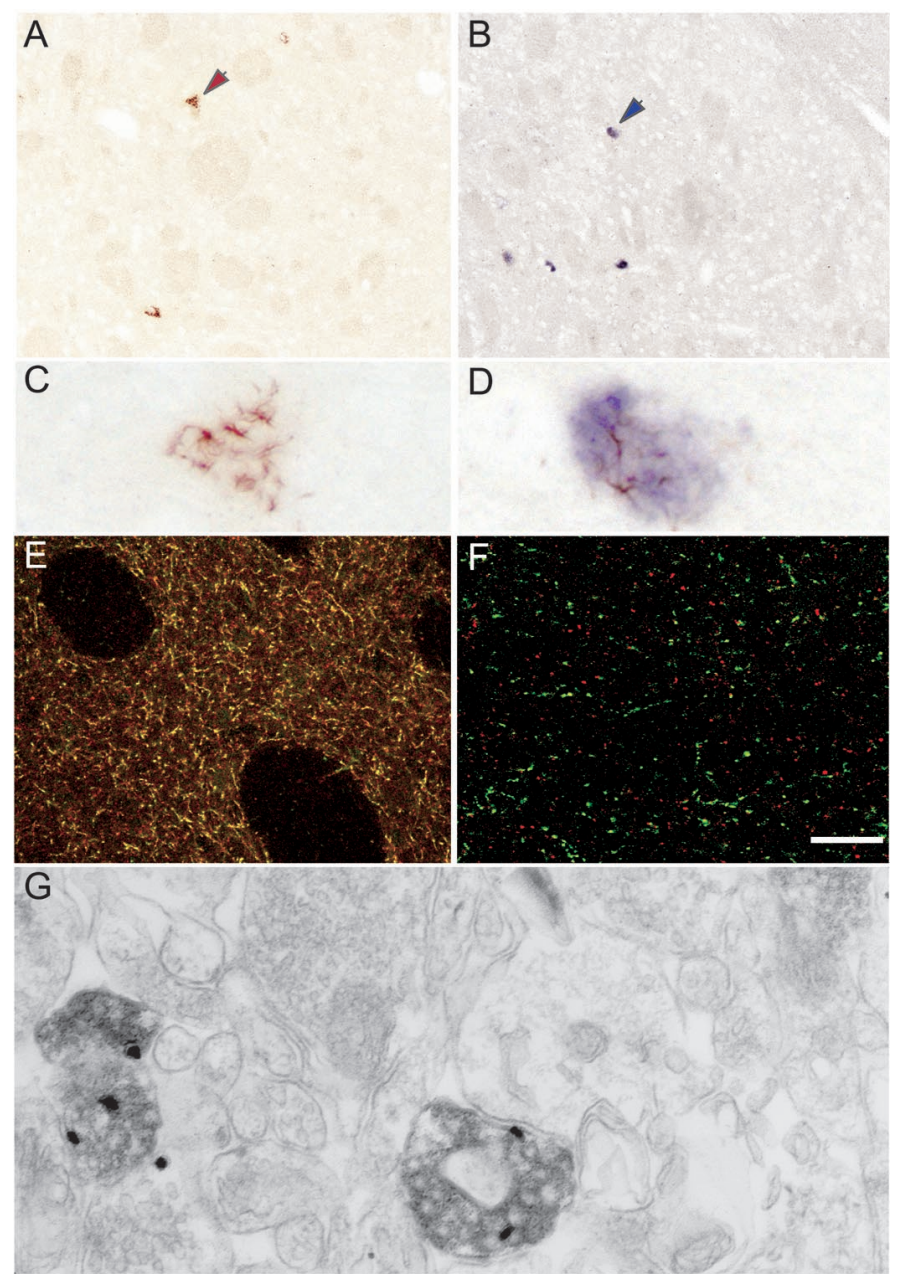

Figure 8. VGLUT3 is colocalized with ChAT and VAChT in the striatum. $A, C$, Cold in situ hybridization with the ChAT cRNA probe alone (in red). $D$, Enlargement of the neuron indicated in $A$ by the red arrow. B, D, Double-labeling in situ hybridization (ChAT in red and VGLUT3 in blue). $D$, The double-labeled neuron indicated by a blue arrow in $B$ has been enlarged. $E, F$, Confocal laser detection of double-immunofluorescence for VGLUT3 (CY3, red fluorescence) and VAChT (fluorescein, green fluorescence) in the striatum $(E)$ or the hippocampus $(F)$. In false colors, overlapping signals appear prominently as yellow-orange. $G$, double detection of VGLUT3 (immunogold) and VAChT (immunoperoxidase) with the electron microscope in the striatum. Scale bars: $A, B, E, F, 50 \mu \mathrm{m} ; C, D, 10 \mu \mathrm{m} ; G, 0.25 \mu \mathrm{m}$.

positive, could be glutamatergic. Because serotoninergic neurons are known to send projections to the substantia nigra, ventral tegmental area, hippocampus, striatum, and cerebral cortex, the VGLUT3 protein present in these regions might thus originate from the raphe nuclei. However, such an hypothesis needs to be investigated further.

\section{DISCUSSION}

\section{VGLUT3 is a novel vesicular glutamate transporter}

Two subtypes of vesicular glutamate transporters, named VGLUT1 and VGLUT2, have been characterized recently. In this study we isolated and functionally characterized VGLUT3, a third member of the family. VGLUT3 represents a novel $\mathrm{H}^{+}$dependent glutamate transporter that is both structurally $(75 \%$ aa identity) and functionally very similar to VGLUT1 and VGLUT2. Because VGLUT3 localizes to synaptic vesicles, as do

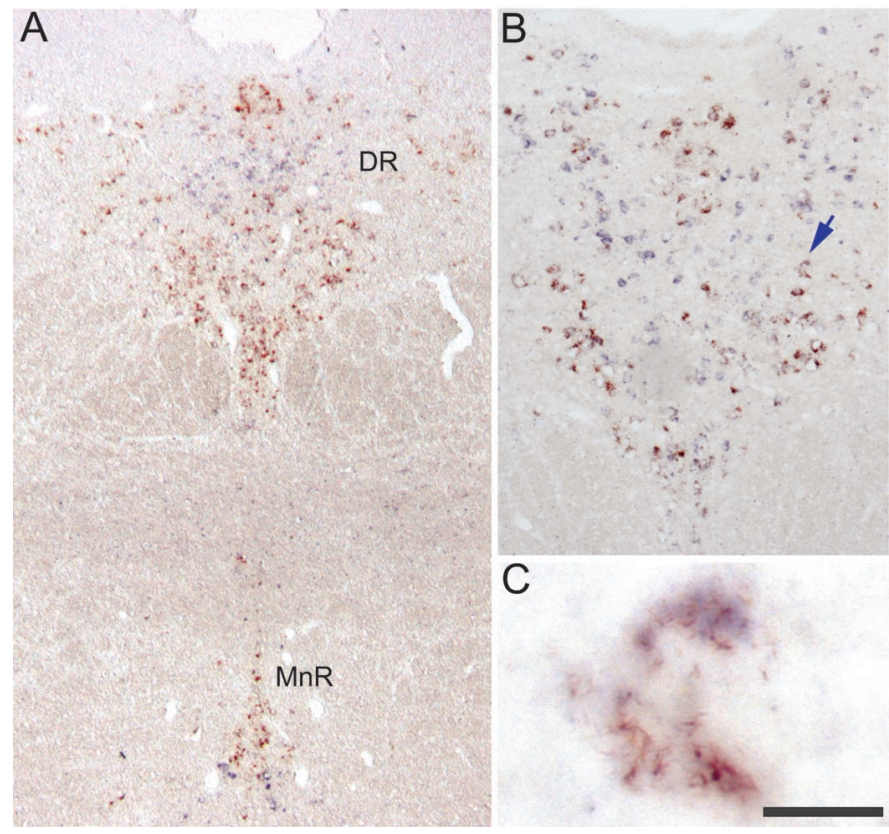

Figure 9. VGLUT3 is colocalized with SERT in the dorsal and medial raphe. The SERT probe is shown in red, and the VGLUT3 probe is shown in blue. $A$ shows the dorsal and medial raphe. $B$, Higher magnification of the dorsal raphe. $C$, Enlargement of double-labeled neurons (indicated in $B$ by a blue arrow). $A$ and $B$ are taken from different sections. $D R$, Dorsal raphe; $M n R$, medial raphe. Scale bars: $A, 300 \mu \mathrm{m} ; B, 100 \mu \mathrm{m} ; C, 10 \mu \mathrm{m}$.

VGLUT1 and VGLUT2, we concluded that it is a novel vesicular glutamate transporter. Our study of its uptake activity revealed subtle differences with VGLUT2, including a slightly increased affinity for glutamate, a lesser sensitivity to Evans Blue, and a higher dependence on the $\Delta \mathrm{pH}$ component of the proton electrochemical gradient. This last property might represent an intrinsic difference between the two transporters, such as the coupling of the VGLUT3-mediated glutamate uptake to the export of more protons (implying a higher loading of vesicles with glutamate). However, we cannot exclude extrinsic factors, such as the localization of VGLUT2 and VGLUT3 in distinct compartments of the BON cells, differing by their lumenal $\mathrm{pH}$. Additional studies are needed to discriminate between these possibilities. A major (eightfold) difference was also observed for the uptake capacity $\left(V_{\max }\right)$ of VGLUT3- and VGLUT2-containing vesicles. However, in the absence of a common ligand enabling us to compare the amounts of both transporters, the issue of whether the slower $V_{\max }$ of VGLUT3 reflects a slower turnover or, merely, a lower expression level will remain open.

\section{VGLUT3 has discrete localization}

Despite the functional similarity to VGLUT1 and VGLUT2, VGLUT3 differs from these isoforms by several unique anatomical features. First, it is expressed in a few scattered neurons of the brain, in contrast to VGLUT1 and VGLUT2, which are massively expressed throughout large areas of the brain. Neurons expressing VGLUT3 are found in brain regions that also contain the VGLUT1 and VGLUT2 mRNAs, such as the cerebral cortex, hippocampus, and brainstem. However, VGLUT3 is the only vesicular glutamate transporter synthesized by striatal neurons. The regional colocalization of VGLUT3 mRNA and protein, together with the double-labeling experiments, demonstrates that VGLUT3 is expressed by striatal and hippocampal interneurons. 
Nonetheless, VGLUT3 also seems to be present in neurons from the raphe region sending long projections (for example to the substantia nigra and the ventral tegmental area). Tracing methods as well as lesion studies should help to clarify this point.

\section{VGLUT3 is present in terminals forming symmetrical and asymmetrical synapses}

Second, at the ultrastructural level, while VGLUT1 and VGLUT2 are exclusively present in terminals forming asymmetrical synapses (Bellocchio et al., 1998; Fremeau et al., 2001; Fujiyama et al., 2001; Herzog et al., 2001; Sakata-Haga et al., 2001), VGLUT3 is found in asymmetrical as well as symmetrical synapses. Therefore, if VGLUT3 is fully functional in vivo, the population of excitatory synapses can no longer be identified by a morphological criterion.

It is tantalizing to consider that VGLUT3-positive symmetrical synapses are cholinergic and serotoninergic while asymmetrical synaptic contacts containing VGLUT3 are glutamatergic. According to Fremeau et al. (2001), all asymmetrical synapses express either VGLUT1 or VGLUT2. It can thus be anticipated that VGLUT3 and VGLUT1 or VGLUT2 are coexpressed in some of these glutamatergic terminals. Such a colocalization is already suspected for VGLUT1 and VGLUT2 (Herzog et al., 2001).

\section{VGLUT3 is present in cholinergic and serotoninergic neurons}

Finally, we report here, for the first time, that cholinergic and serotoninergic neurons express a vesicular glutamate transporter. In this study we have solidly documented the presence VGLUT3 and VAChT in the same terminals. A similar demonstration remains to be established for VGLUT3 and vesicular monoamine transporter type-2 (VMAT2). Whether or not VGLUT3 and VAChT or VMAT2 are found on the same synaptic vesicle is still an open question. If this is the case, then cholinergic and serotoninergic terminals have the potential to store, and thus to release (Takamori et al., 2000, 2001), glutamate in addition to acetylcholine or serotonin. Indeed, concentrations of glutamate above VGLUT3 $K_{\mathrm{M}}$ are achieved in the cytosol of monoaminergic neurons (Kaneko et al., 1990; Danbolt, 2001). Furthermore, the codetection of the excitatory amino acid on one hand and acetylcholine or serotonin on the other hand in the same terminals has already been reported in other brain areas (Clements and Grant, 1990; Waerhaug and Ottersen, 1993; Lavoie and Parent, 1994a,b; Clarke et al., 1996, 1997; Lebrand et al., 1996, 1998; Cases et al., 1998; Hökfelt et al., 2000). The coexpression of the corresponding vesicular transporters thus represents a step further. However, the synaptic corelease of glutamate simultaneously with serotonin or acetylcholine, and its physiological relevance, remains to be formally established. Interestingly, a recent study has reported that dopaminergic neurons can also form asymmetric synaptic specialization and release glutamate in vitro (Sulzer et al., 1998). This study supports the working hypothesis that the corelease of glutamate with other transmitters may be more general than currently suspected.

However, it cannot be ruled out that VGLUT3 is addressed to a different set of functional synaptic vesicles than VAChT/ VMAT2. Alternatively, VGLUT3 could be present in vesicles that do not belong to a readily releasable pool or are in the reserve pool. Moreover, to our knowledge, the presence of glutamate receptor in postsynaptic specialization of the synaptic cleft of cholinergic or serotoninergic neurons has not been docu- mented. If VGLUT3 is in the reserve pool of vesicles or if glutamate ion channel receptors are not present, the physiological function of VGLUT3 will have to be elucidated. Consequently the main challenge for the forthcoming studies on VGLUT3 will be to clearly establish its functionality in monoaminergic neurons.

Cholinergic and serotoninergic neurons are involved in a wide variety of neurological and psychiatric diseases (Kawaguchi et al., 1995; Calabresi et al., 1997; Pollack, 2001). Because of its discrete localization in these neurons, VGLUT3 represents a promising therapeutic target for these pathologies.

\section{REFERENCES}

Aihara Y, Mashima H, Onda H, Hisano S, Kasuya H, Hori T, Yamada S, Tomura H, Yamada Y, Inoue I, Kojima I, Takeda J (2000) Molecular cloning of a novel brain-type $\mathrm{Na}^{+}$-dependent inorganic phosphate cotransporter. J Neurochem 74:2622-2625.

Antunes Bras JM, Epstein AL, Bourgoin S, Hamon M, Cesselin F, Pohl M (1998) Herpes simplex virus 1-mediated transfer of preproenkephalin A in rat dorsal root ganglia. J Neurochem 70:1299-1303.

Bai L, Xu H, Collins JF, Ghishan FK (2001) Molecular and functional analysis of a novel neuronal vesicular glutamate transporter. J Biol Chem 276:36764-36769.

Bellocchio EE, Hu H, Pohorille A, Chan J, Pickel VM, Edwards RH (1998) The localization of the brain-specific inorganic phosphate transporter suggests a specific presynaptic role in glutamatergic transmission. J Neurosci 18:8648-8659.

Bellocchio EE, Reimer RJ, Fremeau Jr RT, Edwards RH (2000) Uptake of glutamate into synaptic vesicles by an inorganic phosphate transporter. Science 289:957-960.

Bernard V, Levey AI, Bloch B (1999) Regulation of the subcellular distribution of $\mathrm{m} 4$ muscarinic acetylcholine receptors in striatal neurons in vivo by the cholinergic environment: evidence for regulation of cell surface receptors by endogenous and exogenous stimulation. J Neurosci 19:10237-10249.

Calabresi P, De Murtas M, Bernardi G (1997) The neostriatum beyond the motor function: experimental and clinical evidence. Neuroscience 78:39-60.

Cases O, Lebrand C, Giros B, Vitalis T, De Maeyer E, Caron MG, Price DJ, Gaspar P, Seif I (1998) Plasma membrane transporters of serotonin, dopamine, and norepinephrine mediate serotonin accumulation in atypical locations in the developing brain of monoamine oxidase A knock-outs. J Neurosci 18:6914-6927.

Clarke NP, Bolam JP, Bevan MD (1996) Glutamate-enriched inputs from the mesopontine tegmentum to the entopeduncular nucleus in the rat. Eur J Neurosci 8:1363-1376.

Clarke NP, Bevan MD, Cozzari C, Hartman BK, Bolam JP (1997) Glutamate-enriched cholinergic synaptic terminals in the entopeduncular nucleus and subthalamic nucleus of the rat. Neuroscience 81:371-385.

Clements JR, Grant S (1990) Glutamate-like immunoreactivity in neurons of the laterodorsal tegmental and pedunculopontine nuclei in the rat. Neurosci Lett 120:70-73.

Danbolt NC (2001) Glutamate uptake. Prog Neurobiol 65:1-105

Davis S, Salin H, Helme-Guizon A, Dumas S, Stephan A, Corbex M, Mallet J, Laroche S (2000) Dysfunctional regulation of $\alpha$ CaM KII and syntaxin 1B transcription after induction of LTP in the aged rat. Eur J Neurosci 12:3276-3282.

Dent JA, Davis MW, Avery L (1997) avr-15 encodes a chloride channel subunit that mediates inhibitory glutamatergic neurotransmission and ivermectin sensitivity in Caenorhabditis elegans. EMBO J 16:5867-5879.

Erickson JD, Varoqui H (2000) Molecular analysis of vesicular amine transporter function and targeting to secretory organelles. FASEB J 14:2450-2458.

Evers BM, Townsend Jr CM, Upp JR, Allen E, Hurlbut SC, Kim SW, Rajaraman S, Singh P, Reubi JC, Thompson JC (1991) Establishment and characterization of a human carcinoid in nude mice and effect of various agents on tumor growth. Gastroenterology 101:303-311.

Fremeau RT, Troyer MD, Pahner I, Nygaard GO, Tran CH, Reimer RJ, Bellocchio EE, Fortin D, Storm-Mathisen J, Edwards RH (2001) The expression of vesicular glutamate transporters defines two classes of excitatory synapse. Neuron 31:247-260.

Fujiyama F, Furuta T, Kaneko T (2001) Immunocytochemical localization of candidates for vesicular glutamate transporters in the rat cerebral cortex. J Comp Neurol 435:379-387.

Gasnier B (2000) The loading of neurotransmitters into synaptic vesicles. Biochimie 82:327-337.

Gilmor ML, Nash NR, Roghani A, Edwards RH, Yi H, Hersch SM, Levey AI (1996) Expression of the putative vesicular acetylcholine transporter in rat brain and localization in cholinergic synaptic vesicles. J Neurosci 16:2179-2190. 
Hayashi M, Otsuka M, Morimoto R, Hirota S, Yatsushiro S, Takeda J, Yamamoto A, Moriyama Y (2001) Differentiation-associated $\mathrm{Na}^{+}$dependent inorganic phosphate cotransporter (DNPI) is a vesicular glutamate transporter in endocrine glutamatergic systems. J Biol Chem 276:43400-43406.

Herzog E, Bellenchi GC, Gras C, Bernard V, Ravassard P, Bedet C, Gasnier B, Giros B, El Mestikawy S (2001) The existence of a second vesicular glutamate transporter specifies subpopulations of glutamatergic neurons. J Neurosci 21:RC181:1-6.

Hisano S, Haga H, Li Z, Tatsumi S, Miyamoto KI, Takeda E, Fukui Y (1997) Immunohistochemical and RT-PCR detection of $\mathrm{Na}^{+}$dependent inorganic phosphate cotransporter $(\mathrm{NaPi}-2)$ in rat brain. Brain Res 772:149-155.

Hisano S, Hoshi K, Ikeda Y, Maruyama D, Kanemoto M, Ichijo H, Kojima I, Takeda J, Nogami H (2000) Regional expression of a gene encoding a neuron-specific $\mathrm{Na}^{+}$-dependent inorganic phosphate cotransporter (DNPI) in the rat forebrain. Brain Res Mol Brain Res 83:34-43.

Hökfelt T, Arvidsson U, Cullheim S, Millhorn D, Nicholas AP, Pieribone V, Seroogy K, Ulfhake B (2000) Multiple messengers in descending serotonin neurons: localization and functional implications. J Chem Neuroanat 18:75-86.

Kaneko T, Akiyama H, Nagatsu I, Mizuno N (1990) Immunohistochemical demonstration of glutaminase in catecholaminergic and serotoninergic neurons of rat brain. Brain Res 507:151-154.

Kawaguchi Y, Wilson CJ, Augood SJ, Emson PC (1995) Striatal interneurones: chemical, physiological, and morphological characterization. Trends Neurosci 18:527-535.

Lavoie B, Parent A (1994a) Pedunculopontine nucleus in the squirrel monkey: distribution of cholinergic and monoaminergic neurons in the mesopontine tegmentum with evidence for the presence of glutamate in cholinergic neurons. J Comp Neurol 344:190-209.

Lavoie B, Parent A (1994b) Pedunculopontine nucleus in the squirrel monkey: cholinergic and glutamatergic projections to the substantia nigra. J Comp Neurol 344:232-241.

Lebrand C, Cases O, Adelbrecht C, Doye A, Alvarez C, El Mestikawy S, Seif I, Gaspar P (1996) Transient uptake and storage of serotonin in developing thalamic neurons. Neuron 17:823-835.

Lebrand C, Cases O, Wehrle R, Blakely RD, Edwards RH, Gaspar P (1998) Transient developmental expression of monoamine transporters in the rodent forebrain. J Comp Neurol 401:506-524.

Lee RY, Sawin ER, Chalfie M, Horvitz HR, Avery L (1999) EAT-4, a homolog of a mammalian sodium-dependent inorganic phosphate cotransporter, is necessary for glutamatergic neurotransmission in $\mathrm{Cae}$ norhabditis elegans. J Neurosci 19:159-167.

Ni B, Rosteck Jr PR, Nadi NS, Paul SM (1994) Cloning and expression of a cDNA encoding a brain-specific $\mathrm{Na}^{+}$-dependent inorganic phosphate cotransporter. Proc Natl Acad Sci USA 91:5607-5611.

Ni B, Wu X, Yan GM, Wang J, Paul SM (1995) Regional expression and cellular localization of the $\mathrm{Na}^{+}$-dependent inorganic phosphate cotransporter of rat brain. J Neurosci 15:5789-5799.

Ozkan ED, Ueda T (1998) Glutamate transport and storage in synaptic vesicles. Jpn J Pharmacol 77:1-10.

Pollack AE (2001) Anatomy, physiology, and pharmacology of the basal ganglia. Neurol Clin 19:523-534.

Reimer RJ, Fon EA, Edwards RH (1998) Vesicular neurotransmitter transport and the presynaptic regulation of quantal size. Curr Opin Neurobiol 8:405-412.

Roghani A, Shirzadi A, Kohan SA, Edwards RH, Butcher LL (1996) Differential distribution of the putative vesicular transporter for acetylcholine in the rat central nervous system. Brain Res Mol Brain Res 43:65-76.

Roseth S, Fykse EM, Fonnum F (1995) Uptake of L-glutamate into rat brain synaptic vesicles: effect of inhibitors that bind specifically to the glutamate transporter. J Neurochem 65:96-103.

Sakata-Haga H, Kanemoto M, Maruyama D, Hoshi K, Mogi K, Narita M, Okado N, Ikeda Y, Nogami H, Fukui Y, Kojima I, Takeda J, Hisano S (2001) Differential localization and colocalization of two neuron-types of sodium-dependent inorganic phosphate cotransporters in rat forebrain. Brain Res 902:143-155.

Shepherd GM (1998) The synaptic organization of the brain, Ed 4. New York: Oxford UP

Sulzer D, Joyce MP, Lin L, Geldwert D, Haber SN, Hattori T, Rayport S (1998) Dopamine neurons make glutamatergic synapses in vitro. J Neurosci 18:4588-4602.

Takamori S, Rhee JS, Rosenmund C, Jahn R (2000) Identification of a vesicular glutamate transporter that defines a glutamatergic phenotype in neurons. Nature 407:189-194.

Takamori S, Rhee JS, Rosenmund C, Jahn R (2001) Identification of differentiation-associated brain-specific phosphate transporter as a second vesicular glutamate transporter (VGLUT2). J Neurosci 21:RC182:1-6.

Varoqui H, Schafer MK, Zhu H, Weihe E, Erickson JD (2002) Identification of the differentiation-associated $\mathrm{Na}^{+} / \mathrm{PI}$ transporter as a novel vesicular glutamate transporter expressed in a distinct set of glutamatergic synapses. J Neurosci 22:142-155.

Waerhaug O, Ottersen OP (1993) Demonstration of glutamate-like immunoreactivity at rat neuromuscular junctions by quantitative electron microscopic immunocytochemistry. Anat Embryol (Berl) 188:501-513.

Weihe E, Tao-Cheng JH, Schafer MK, Erickson JD, Eiden LE (1996) Visualization of the vesicular acetylcholine transporter in cholinergic nerve terminals and its targeting to a specific population of small synaptic vesicles. Proc Natl Acad Sci USA 93:3547-3552. 